Rosanna Cerbo

Maria Pia Prudenzano

Piero Barbanti

Mariantonietta Savarese

Virgilio Gallai • Andrea Alberti

Maria Nicolodi • Stefania Canova

Gennaro Bussone • Domenico D'Amico

Giuseppe Libro • Licia Grazzi

Franco Granella

Giorgio Zanchin

Giorgio Sandrini

Anna Pia Verri

Giorgio Nider

Giuliano Relja
R. Cerbo (凶) • P. Barbanti

Department of Neurological Sciences

University of Rome La Sapienza

Viale dell'Università 30, I-00185 Rome, Italy

e-mail: rosanna.cerbo@uniroma1.it

Tel.: +39-06-49914732/34

Fax: +39-06-4457376

The affiliations of the other authors are listed at the end of the article

\section{The importance of anxiety and depression as factors in chronicization of primary headaches}

\begin{abstract}
A multicenter study was carried out in 10 Italian headache centers to investigate the prevalence of anxiety and depression in patients with chronic daily headache (CDH). The study investigated 219 patients (171 F; $48 \mathrm{M}$ ) including 53 cases of chronic tension-type headache (CTTH), 99 cases of CTTH + migraine and 67 cases of transformed migraine (TM). The type of headache diagnosed at the beginning was episodic tension-type headache (ETTH, $\mathrm{n}=32$ ), ETTH + migraine $(\mathrm{n}=2), \mathrm{CDH}$ ab initio $(\mathrm{n}=22)$, migraine with/without aura $(\mathrm{n}=151)$, not classifiable migraine $(\mathrm{n}=7)$ and not classifiable headache $(\mathrm{n}=3)$. The assessment of anxiety
\end{abstract}

and depression was carried out using a Zung self-rating scale for anxiety (Zung A) and for depression (Zung D). The results show that anxiety and depression levels, in each group, were related to sex $(\mathrm{F}>\mathrm{M})$. Anxiety, but not depression, was related to the length of chronicization process. Anxiety and depression did not correlate with type of headache at onset, with ongoing headache or, surprisingly, with the abuse of anti-inflammatory drugs. These data suggest the chronicization is a biological and psychological trait.

Key words Chronic daily headache • Anxiety $\cdot$ Depression $\cdot$ Overuse of analgesics $\cdot$ Zung scale

\section{Introduction}

The association between primary headache and mood disorders, in particular anxiety and depression, is well documented in literature [1-3]. Several population-based studies have demonstrated an association between migraine and major depression and anxiety disorders, panic disorder and other psychiatric disturbances [4-8]. In particular, a high comorbidity with psychiatric disorders has been observed in patients with chronic daily headache. Nonetheless, an association between anxiety and depression in patients with chronic tension-type headache has been observed, but not in those with episodic tension-type headache [9]. Depression is also found in almost $80 \%$ of patients with transformed migraine [10-12].
The biological relationship of chronic primary headache and psychiatric comorbidity still remains to be clarified. Several are the interpretations of such associations: depression may be a result, and not a cause, of chronic pain; headache may even be interpreted as a somatic evidence of a psychopathologic primary process; finally, the associations among anxiety, depression and chronic headaches may underlie a common biologic pathway.

It is also well known that the abuse of anti-inflammatory drugs may be a relevant factor in the chronicization of episodic headaches. Patients with drug abuse commonly appear more depressed and anxious than those without drug abuse. The Italian Collaborative Group for the Study of Psychopathological Factors in Primary Headaches has previously investigated psychiatric aspects in a wide sample of 
patients with migraine with or without aura and in patients with episodic or chronic tension-type headache [13, 14].

The aim of this study was to evaluate the importance of factors such as anxiety and depression in patients with chronic headache. In particular, we wanted to investigate the relationships among anxiety, depression and anti-inflammatory drug abuse.

\section{Patients and methods}

The study enrolled 219 outpatients, including 171 women and 48 men, referred to Italian tertiary care headache centers and affected by chronic daily headache $(\mathrm{CDH})$. Mean age was $47.71 \pm 12.93$ years (women, $43.6 \pm 12.7$; men, $39.6 \pm 13.7$ years), mean duration of headache was $22.2 \pm 13.7$ years and the duration of chronic headache was $6.9 \pm 7.6$ years. The diagnosis of initial headache was episodic tension-type headache in 32 patients $(21 \mathrm{~F}, 11 \mathrm{M})$, episodic tension-type headache and migraine in 2 female patients, ab initio chronic headache in 22 patients $(12 \mathrm{~F}, 10 \mathrm{M})$, migraine with aura in 10 patients ( $8 \mathrm{~F}, 2 \mathrm{M})$, migraine without aura in 141 patients $(117 \mathrm{~F}, 24 \mathrm{M})$, not classifiable migraine in 7 patients $(6 \mathrm{~F}$, $1 \mathrm{M}$ ), and not classifiable headache in 3 female patients. For another 2 female patients, no information on initial headache was available. The form of chronic daily headache $(\mathrm{CDH})$ at moment of observation was chronic tension-type headache (TTH) in 53 patients (33 F, $20 \mathrm{M}$ ), chronic tension-type headache and migraine in 99 patients $(81 \mathrm{~F}, 18 \mathrm{M})$, and transformed migraine in 67 patients $(57 \mathrm{~F}, 10 \mathrm{M})$. The assessment of anxiety and depression was carried out using a Zung self-rating scale for anxiety (Zung A cut-off $=40$ ) and for depression (Zung D cut-off $=41$ ).

Statistical analysis was performed using the variance analysis of Kruskal-Wallis and ANOVA for current and past type of headache. The comparison between men and women was performed using Mann-Whitney $\mathrm{U}$ test and $t$ test for unpaired data.

\section{Results}

Women showed higher Zung anxiety and depression values with respect to men in all $\mathrm{CDH}$ groups (Tables 1,2).

Anxiety and depression values were higher in older patients and in patients with longer duration of illness (data not shown). The age at onset of the chronic headache was positively correlated with anxiety and depression values (Table 3). A positive correlation between duration of chronic headache was observed for anxiety, but not for depression (Table 3). No correlation was observed between Zung scales for anxiety and depression and previous type of headache or the age at onset.

No difference was found either in anxiety or in depression scores between overuse and no overuse groups within both sexes and within the overall sample (Table 4).

Table 1 Anxiety values (Zung scale) in patients with $\mathrm{CDH}$, according to headache diagnosis at observation time

\begin{tabular}{|c|c|c|c|c|c|c|}
\hline & \multicolumn{2}{|c|}{$\operatorname{Men}(n=171)$} & \multicolumn{2}{|r|}{ Women $(n=48)$} & \multicolumn{2}{|c|}{ Total $(n=219)$} \\
\hline & $\mathrm{n}$ & Zung score & $\mathrm{n}$ & Zung score & $\mathrm{n}$ & Zung score \\
\hline СТTH & 20 & $37.2 \pm 8.7 * *$ & 33 & $44.3 \pm 9.2 * *$ & 53 & $41.6 \pm 9.6 *$ \\
\hline $\mathrm{CTTH}+\mathrm{M}$ & 18 & $36.1 \pm 6.5 * *$ & 81 & $45.1 \pm 9.2 * *$ & 99 & $43.3 \pm 9.5 *$ \\
\hline $\mathrm{TM}$ & 10 & $37.3 \pm 9.2 * *$ & 57 & $46.5 \pm 8.3 * *$ & 67 & $45.1 \pm 9.0 *$ \\
\hline
\end{tabular}

** $p<0.05$ (Mann Whitney test) (M vs. F)

$* p=$ n.s. (variance analysis)

$C D H$, chronic daily headache; $C T T H$, chronic tension-type headache; $M$, migraine; $T M$, transformed migraine

Table 2 Depression values (Zung scale) in patients with $\mathrm{CDH}$, according to headache diagnosis at observation time

\begin{tabular}{lccc}
\hline & Men $(\mathrm{n}=171)$ & Women $(\mathrm{n}=48)$ & Total $(\mathrm{n}=219)$ \\
\hline CTTH & $39.6 \pm 8.9 * *$ & $46.1 \pm 9.9 * *$ & $43.7 \pm 10.0 *$ \\
CTTH + M & $36.2 \pm 7.5 * *$ & $44.5 \pm 10.0 * *$ & $43.0 \pm 10.1 *$ \\
TM & $39.7 \pm 11.6^{* *}$ & $46.3 \pm 10.4 *$ & $49.3 \pm 10.8 *$ \\
\hline
\end{tabular}

** $p<0.05$ (Mann Whitney test) (M vs. F)

$* p=$ n.s. (variance analysis)

$C D H$, chronic daily headache; $C T T H$, chronic tension-type headache; $M$, migraine; $T M$, transformed migraine 
Table 3 Correlation between clinical features and anxiety and depression scores on Zung scales

\begin{tabular}{lll}
\hline & \multicolumn{1}{c}{ Zung anxiety } & Zung depression \\
\hline Age & $\mathrm{r}=0.244(p=0.001)$ & $\mathrm{r}=0.209(p=0.002)$ \\
Age at onset & $\mathrm{r}=0.049(p=\mathrm{n} . \mathrm{s})$. & $\mathrm{r}=0.008(p=\mathrm{n} . \mathrm{s})$. \\
Age at onset of chronic headache & $\mathrm{r}=0.168(p=0.01)$ & $\mathrm{r}=0.140(p=0.04)$ \\
Headache duration & $\mathrm{r}=0.193(p=0.004)$ & $\mathrm{r}=0.203(p=0.003)$ \\
Duration of chronic headache & $\mathrm{r}=0.1423(p=0.03)$ & $\mathrm{r}=0.129(p=\mathrm{n} . \mathrm{s})$. \\
\hline
\end{tabular}

r, Pearson's coefficient

Table 4 Anxiety and depresion scores according to Zung in patients with or without abuse of anti-inflammatory drugs. Values in parentheses are standard deviations

\begin{tabular}{|c|c|c|c|c|c|c|c|c|c|}
\hline \multirow{4}{*}{$\begin{array}{l}\text { Zung Depression } \\
\text { Zung Anxiety }\end{array}$} & \multicolumn{5}{|c|}{ Overuse $(n=142)$} & \multicolumn{4}{|c|}{ No overuse $(\mathrm{n}=77)$} \\
\hline & \multicolumn{2}{|c|}{$\mathrm{F}(\mathrm{n}=118)$} & \multicolumn{2}{|c|}{$M(n=24)$} & Total & $\mathrm{F}(\mathrm{n}=53)$ & \multicolumn{2}{|c|}{$M(n=24)$} & \multirow{2}{*}{$\frac{\text { Total }}{43.2(10.3)}$} \\
\hline & 45.2 & $(10.1)$ & 39.6 & $(10.5)$ & $44.2 \quad(10.3)$ & $45.9(10.4)$ & 37.2 & (7.3) & \\
\hline & 45.7 & $(9.1)$ & 37.7 & $(9.0)$ & $44.3 \quad(9.5)$ & $44.9 \quad(8.5)$ & 35.2 & (6.7) & $41.9 \quad(9.1)$ \\
\hline
\end{tabular}

\section{Discussion}

Several are the factors involved in the chronicization of an episodic primary headache: abnormal personality profiles, repeated external psychosocial stressors, the prolonged administration of symptomatic drugs and the onset of other illnesses. Psychological aspects are relevant, but it is not yet known if their actual role is in causing the chronicization or if they represent the effect of chronic pain. The Italian Collaborative Group for the Study of Psychopathological Factors in Primary Headaches observed that at least one psychosocial stress event or a psychiatric disorder was detected in $66.2 \%$ of patients with migraine with or without aura and in $84.8 \%$ of patients with tension-type headache [13, 14].

In particular, in TTH patients anxiety was the most common psychiatric diagnosis $(53.4 \%)$, either alone $(45.6 \%)$ or in combination with depression $(36.2 \%)$. The prevalence of anxiety was similar in episodic $(50.9 \%)$ and chronic $(55.9 \%)$ TTH patients. Depression, vice versa, was found in $36.8 \%$ of patients, and was significantly higher in the CTTH group ( $44.9 \%$ vs. $28.7 \%, p=0.02$, Fisher's exact test) [13].

The present study confirmed that in $\mathrm{CDH}$, anxiety and depression are more frequent in patients with a longer history of headache, considering both the episodic and the chronic periods. In all the groups examined (ongoing headache type, headache type at onset, abuse of anti-inflammatory drugs), anxiety and depression scores were higher in women than in men. This result confirms previous observations by Mitsikostas and Thomas [15], who observed higher scores on the Hamilton scale for anxiety and depression in relation to gender (twice as common in women than in men). On the contrary, Ho and coworkers [16] observed no differences between women and men in the correlation of the various forms of primary headache with depression. In a previous study of our group, no gender differences in the occurence of anxiety and depression were observed, either in episodic or in chronic TTH patients [13].

Moreover, the high anxiety and depression rates in our study are not related to the headache at onset. An interesting result is represented by the fact that depression scores are not related to the duration of chronic headache. These results suggest that depression should not be considered the result of headache chronicization, but a peculiar aspect of the psychologic and biologic features of each patient.

A surprising result of our study is that we did not find a relation between the high anxiety and depression scores and the abuse of analgesics. A nociceptive receptorial dysregulation has been hypothesized in patients with primary headache and anti-inflammatory drug overuse. These alteration may be genetically determined. In fact, in a large number of cases, a familiar history of drug abuse has been observed. Abuse may then be related to a biological trait, with serotoninergic and dopaminergic disturbances. In alcoholics, a clear-cut genetic influence has been observed, and the disease has been linked to a specific polymorphism of the D2 receptor called TAQ1 [17]. The fact that depression is not related to the length of the chronicization and that neither anxiety nor depression are related to drug abuse led us to hypothesize that tendency to chronicization represents a biological and psychological trait. 
Migraine and tension-type headache, with different clinical histories and epidemiology, share individual predisposition, generally with familiar distribution, where external factors are crucial in causing the headache attack. As for tension-type headache pathogenesis, the presence of an individual predisposition seems to be specific for the chronic type [18]. It is also interesting to note that the polymorphism for $\mathrm{NcoI} \mathrm{C} / \mathrm{C}$ of D2 receptor is predominant in patients with migraine with aura, anxiety, depression, panic attacks and phobias [19]. We may then assume that the chronicization of episodic headaches is predetermined by biological and psychological traits. In other words, one is born to be a chronic sufferer just as a migraineur is.

M.P. Prudenzano • M. Savarese

Neurological Clinic I, University of Bari, Bari, Italy

V. Gallai • A. Alberti

Neurological Clinic,

University of Perugia, Perugia, Italy
M. Nicolodi $•$ S. Canova

Interuniversity Centre of Neurochemistry and Clinical

Pharmacology of Primary Headache,

Florence University, Florence, Italy

G. Bussone • D. D’Amico • G. Libro • L. Grazzi

C. Besta National Neurological Institute,

Milan, Italy

F. Granella

Institute of Neurology,

University of Parma, Parma, Italy

G. Zanchin

Neurological Clinic, University of Padua,

Padua, Italy

G. Sandrini • A.P. Verri

IRCCS Mondino, University of Pavia, Pavia, Italy

G. Nider • G. Relja

Ospedale Maggiore, Trieste, Italy

\section{References}

1. Wolf HG (1937) Personality features and reactions of subjects with migraine. Archives of Neurology and of General Psychiatry 37:895-921

2. Breslau N, Davis GC (1993) Migraine, physical health and psychiatric disorders: a prospective epidemiological study of young adults. J Psychiatric Res 27(2):211-221

3. Silberstein SD, Lipton RB, Breslau N (1995) Migraine: association with personality characteristics and psychopathology. Cephalalgia 15:1-15

4. Merikangas KR, Stevens DE, Angst J (1993) Headache and personality: results of a community sample of young adults. J Psychiat Res 27:187-196

5. Merikangas KR, Angst J, Isler $H$ (1990) Migraine and psychopathology. Results of the Zurich cohort study of young adults. Arch Gen Psychiatry 47:849-853

6. Breslau N. Davis GC, Schultz LR et al (1994) Migraine and major depression: a longitudinal study. Headache 7:387-393

7. Stewart WF, Linet MS, Celentano DD (1989) Migraine headaches and panic attacks. Psychosom Med 51:559-569
8. Breslau N, Davis GC (1992) Migraine, major depression and panic disorder: a prospective epidemiologic study of young adults. Cephalalgia 12:85-89

9. Merikangas KR, Angst J (1992) Migraine and psychopathology: epidemiologic and genetic aspects. Clin Neuropharmacol 1:275A

10. Mathew NT (1991) Chronic daily headache; clinical features and natural history. In: Nappi G, Bono G, Sandrini G, Martignoni E, Micieli G (eds) Headache and depression: serotonin pathways as a common clue. Raven, New York, pp 49-58

11. Mathew NT (1990) Drug-induced headache. Clin Neuropharm 8: 903-912

12. Saper JR (1987) Ergotamine dependence - a review. Headache 27:435-438

13. The Italian Collaborative Group for the Study of Psychopathological Factors in Primary Headaches (1999) Psychiatric comorbidity and psychosocial stress in patients with tension-type headache from headache centers in Italy, Cephalalgia 19: 159-164
14. Puca FM and the Italian Collaborative Group for the Study of

Psychopathological Factors in Primary Headaches (2000) Psychological and social stressors and psychiatric comorbidity in patients with migraine without aura from headache centers in Italy: a comparison with tension-type headache patients. J Headache Pain 1:15-25

15. Mitsikostas DD, Thomas AM (1999) Comorbidity of headaches and depression. Cephalalgia 19:211-217

16. Ho K-H, Ong BKC, Lee S-C (1997) Headache and self-assessed depression scores in Singapore University undergraduates. Headache 37:26-30

17. Blum K, Noble EP, Sheridan PJ et al (1993) Genetic predisposition in alcoholics: association of the D2 dopamine receptor TaqI B1 RFLP with severe alcoholics. Alcohol 10(1):59-67

18. Schoenen J, Jamart B, Gerard P et al (1987) Exteroceptive suppression of temporalis muscle activity in chronic headache. Neurology 37:1834-1836

19. Peroutka SJ, Price SC, Wilhoit TL, Jones KW (1998) Comorbid migraine with aura, anxiety, and depression is associated with dopamine D2 receptors (DRD2) NcoI alleles. Mol Med 4(1):14-21 